\title{
Colorectal Cancer Epidemic in Korea
}

\author{
Dong-Hyun Kim \\ Department of Social and Preventive Medicine, Hallym University College of Medicine, Chuncheon, Korea
}

Colorectal cancer is the third most common cancer in men $(663,000$ cases, $10.0 \%$ of the total) and the second in women (571,000 cases, $9.4 \%$ of the total) worldwide. Colorectal cancer appears to have rapidly increased over the past two decades in some Asian countries, including Taiwan, Japan, and Korea, that were previously thought to be low risk areas. In Korea, age-adjusted mortality rates for colorectal cancer in 2011 were 4.7 and 3.3 times higher than those in 1983 for men and women, respectively (Fig. 1). Moreover, the increased mortality due to colorectal cancer over the same period appears to be steeper than those due to lung cancer in both men and women while mortalities due to cancers of the stomach and the liver have steadily declined [1]. The mortality rate due to colorectal cancer during the same time period sharply increased by 7.6- and 5.7fold among elderly men and women aged 65 or older, respectively, and by 2.8- and 2.2-fold among middle-aged men and women, respectively (Fig. 2). Based on Central Cancer Registry Program, which generates nationwide cancer incidence rates, in Korea [2], colorectal cancer was the second most common cancer in men $(62.5$ per 100,000) and the third in women $(40.8$ per $100,000)$ in 2010 (Fig. 3).

A comparison of trends in the incidence rates for major cancers during the past decade showed that in Korea, the age-adjusted incidence rates of colorectal cancer between 1999 and 2010 markedly increased from 27 to 49.8 per 100,000 (annual change, $6.3 \%$ ) in men and from 17.1 to 26.4 per 100,000 in women (annual change, 4.7\%) while those of stomach, liver, lung, and cervical cancer decreased (Fig. 4). When compared with other Asian countries [3], the age-adjusted colorectal cancer incidence in men ranked the highest in Korea, followed by Israel, Japan, and Singapore, and that in women ranked 4th highest among Asian countries (Fig. 5). Similarly, high colorec-

Correspondence to: Dong-Hyun Kim, M.D.

Department of Social and Preventive Medicine, Hallym University College of Medicine, 1 Hallimdaehak-gil, Chuncheon 200-702, Korea

Tel: +82-33-248-2660, Fax: +82-33-256-1675

E-mail:dhkims@hallym.ac.kr

(C) 2013 The Korean Society of Coloproctology

This is an open-access article distributed under the terms of the Creative Commons Attribution NonCommercial License (http://creativecommons.org/licenses/by-nc/3.0) which permits unrestricted noncommercial use, distribution, and reproduction in any medium, provided the original work is properly cited. tal cancer incidence rates among Koreans are also reported in the California Regional Cancer Registry. Recent rates of colorectal cancer among Korean immigrants living in that area have been approaching those recorded for Japanese and non-Hispanic whites [4].

All the data suggest that colorectal cancer is epidemic among Koreans, especially among the elderly. Environmental factors, characterized by a western lifestyle, seem to be closely related to the increased risk of colorectal cancer. Higher intakes of red and processed meat, a lack of physical activity, obesity, and alcohol drinking have been suggested to be risk factors for colorectal cancer in the numerous epidemiologic studies [5], while higher intakes of dietary fiber, green leafy vegetables, some micronutrients abundant in vegetables and fruits, such as folate, and calcium were reported to be protective factors. Because many dietand lifestyle-related factors for colorectal cancer are modifiable, it is urgently needed to set up comprehensive primary prevention program against colorectal cancer to effectively cope with the rapidly increasing cancer in Korea. Several specific associations have also been observed between genetic polymorphisms and colorectal cancer. Moreover, environmental factors and related genetic predispositions have been reported to work in concert in colorectal cancer development. For example, the protective effect of the homozygous variant TT form of the methylenetetrahydrofolate reductase (MTHFR) C677T genotype on the risk of colorectal cancer seems to be modified by the level of methyl diets, i.e., by folate, which has a protective effect, or conversely by alcohol. Recommendation of higher intake of folate and lower intake of alcohol to the target population, especially those with TT genotype of MTHFR, may be an effective preventive approach against colorectal cancer [6]. Further studies are needed to clarify how these suggested environmental factors interact with genetic factors to modify the risk of colorectal cancer among Koreans and to develop more tailored preventive strategies applicable to Koreans.

\section{CONFLICT OF INTEREST}

No potential conflict of interest relevant to this article was reported. 
Coloproctology
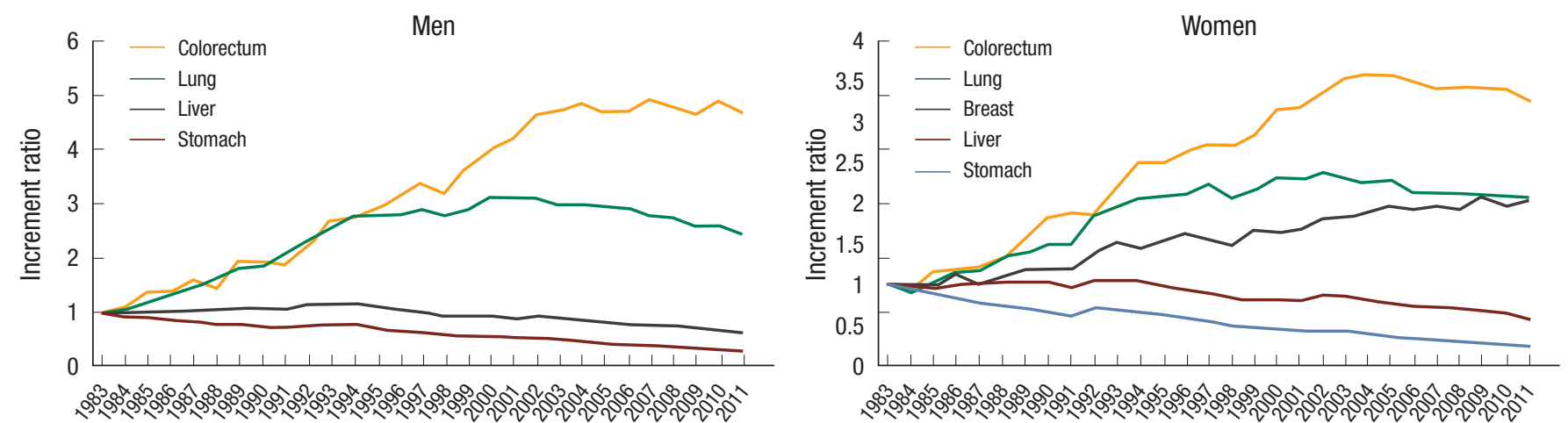

Fig. 1. Increment ratio of age-adjusted a mortality rates compared with that of 1983 for major cancers among men and women, 1983-2011, Korea (adapted from Statistics Korea ${ }^{1)}$ ). ${ }^{a}$ Age was adjusted to the 2000 population census in Korea.
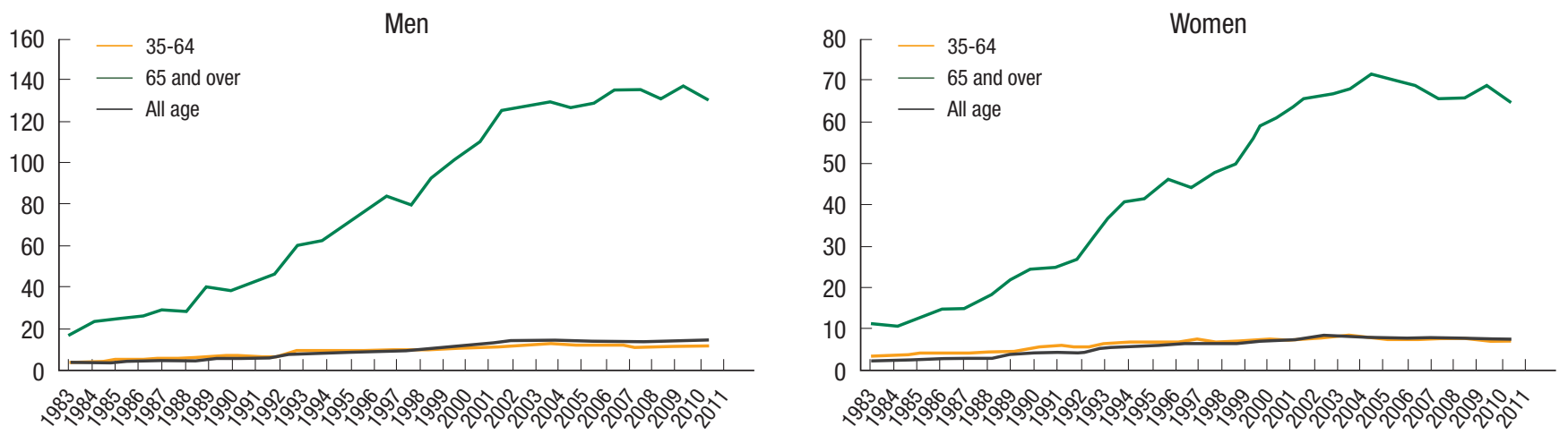

Fig. 2. Trend of the age-adjusted a mortality rate for colorectal cancer by age group in Korea, 1983-2011 (adapted from Statistics Korea ${ }^{1)}$ ). ${ }^{a}$ Age was adjusted to the 2000 population census in Korea.
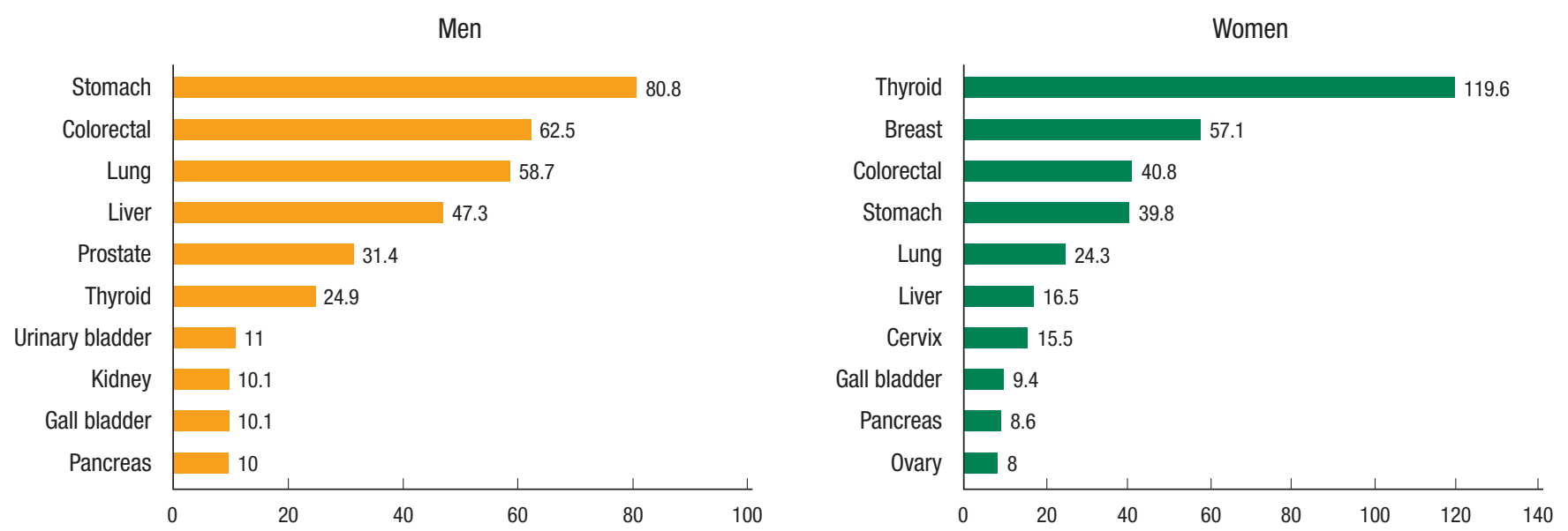

Fig. 3. Crude incidence rates for major cancers in Korea, 2010 (adapted from National Cancer Center ${ }^{2)}$ ). 
Men

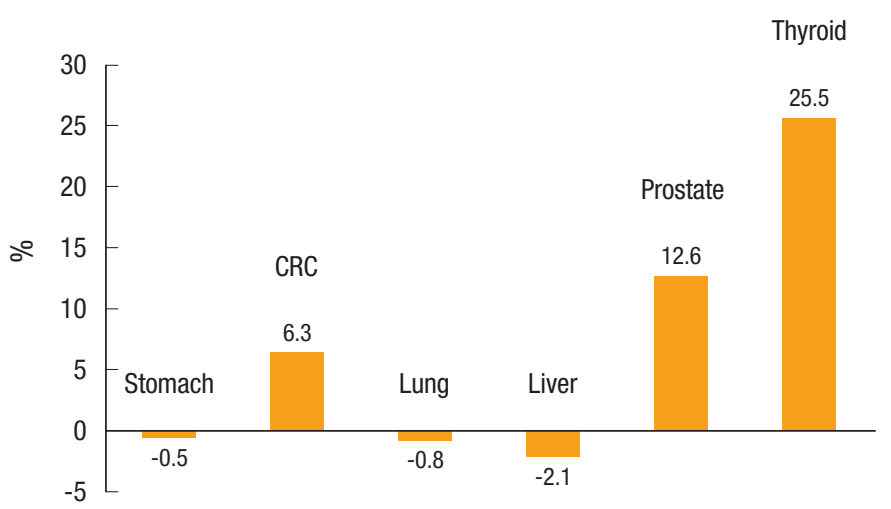

Women

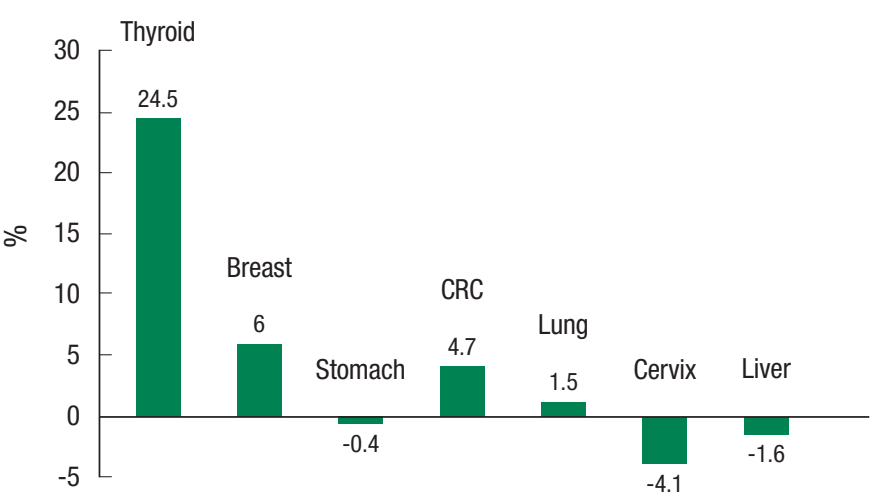

Fig. 4. Change of age-adjusted a incidence rates of major cancers in Korea, 1999 to 2010 (adapted from National Cancer Center ${ }^{2}$ ). ${ }^{a}$ Age was adjusted to the 2000 population census in Korea (adapted from Statistics Korea ${ }^{1)}$ ). CRC, colorectal cancer.
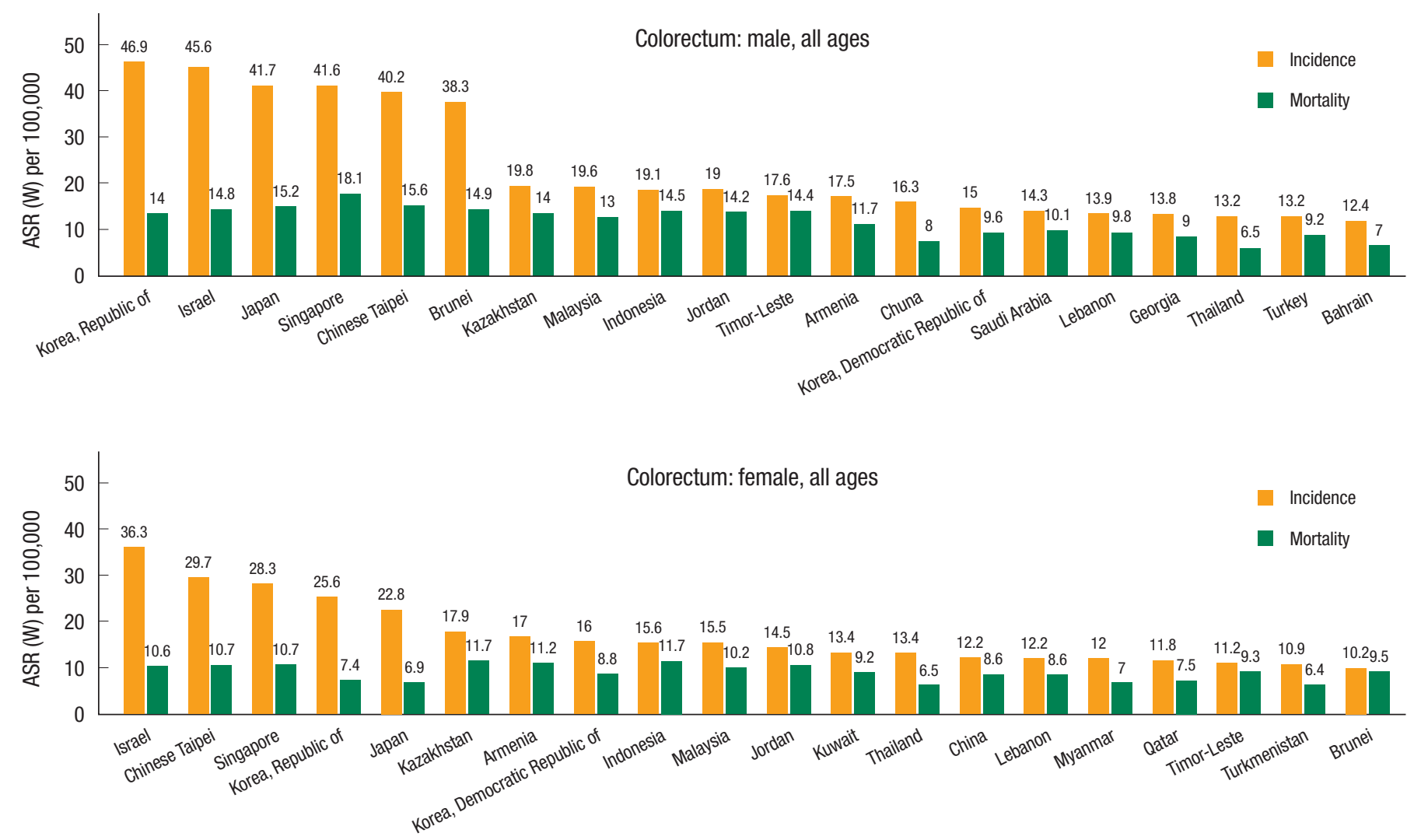

Fig. 5. Age-adjusted colorectal cancer incidence rates and mortality rates among Asian countries, 2008 (adapted from International Agency for Research on Cancer $^{3)}$ ). ASR, age-standardized incidence rate. 


\section{Coloproctology Dong-Hyun Kim}

\section{REFERENCES}

1. Statistics Korea. Korean Statistical Information Service. Deaths and death rates By cause (236 item)/By sex/By age (five year age) [Internet]. Daejeon: Statistics Korea, Korean Statistical Information Service, c2010 [cited 2013 Feb 22]. Available from: http://kosis.kr/eng/database/database_001000.jsp?listid=D\&subtitle= Health/Society/Welfare.

2. National Cancer Center. National Cancer Registration Program [Internet]. Goyang: National Cancer Center; [cited 2013 Feb 22]. Available from: http://www.ncc.re.kr/english/programs/progarms02.jsp.

3. International Agency for Research on Cancer. Cancer incidence in five continents Vol. IX [Internet]. Lyon: International Agency for Research on Cancer; c2013 [cited 2013 Feb 22]. Available from: http://www.iarc.fr/en/publications/pdfs-online/epi/sp160/ index.php.

4. McCracken M, Olsen M, Chen MS Jr, Jemal A, Thun M, Cokkinides V, et al. Cancer incidence, mortality, and associated risk factors among Asian Americans of Chinese, Filipino, Vietnamese, Korean, and Japanese ethnicities. CA Cancer J Clin 2007;57:190205.

5. World Cancer Research Fund/American Institute of Cancer Research. Food, nutrition, physical activity and the prevention of cancer: a global perspective. Washington DC: American Institute of Cancer Research; 2007.

6. Kim DH. The interactive effect of methyl-group diet and polymorphism of methylenetetrahydrofolate reductase on the risk of colorectal cancer. Mutat Res 2007;622:14-8. 